across the plane at a given longitude and frequency gives the 'vertical' distribution of the neutral hydrogen at a particular place in the galaxy. In particular, the galactic latitude at which peak brightness occurs gives a point on the 'equatorial surface' of the layer of neutral hydrogen.

Thus we can delineate a section through the galaxy for each line of sight; the observations give the variation of galactic latitude with radial velocity, from which we can derive a curve showing the height of the equatorial surface as a function of the distance.

The hydrogen is confined to a thin layer (about 250 parsecs between half-density points) in the galactic disk, and observations of this type provide the best method for a new determination of the galactic plane, the position of which is under review by the International Astronomical Union.

A relief map of the galaxy, built up from the Sydney observations, is shown in Fig. 2a. This picture is based on the same velocity model as the spiral diagram, and again it has been drawn without making allowance for random motions ; but the main features should not be changed by later modifications.

The gas layer is found to be remarkably flat in the inner parts of the galaxy. We have called the mean plane in this region the 'principal plane of the galaxy'. (This plane does not necessarily pass through the Sun.) For the present purpose, we have used an approximation to the principal plane as the reference level in Fig. 2. This approximation is a plane which contains the Sun, and slopes downwards from the conventional 'galactic plane' at an angle of $1.5^{\circ}$ towards the galactic centre at longitude $327 \cdot 5^{\circ}$.

In the outer regions, the gas layer shows a sys. tematic distortion, with a twist downwards on the left of the picture and upwards on the right. The effect can be seen more clearly in the cross-section of the galaxy given in Fig. $2 b$. The distortion appears to be a large-scale property of the galaxy, as the height of the gas layer varies fairly regularly, with little relationship to the pattern of spiral arms. This effect has also been noticed by Burke ${ }^{6}$.

The downward deviation is greatest in the region nearest the Magellanic Clouds, which are below the plane in the position indicated; the upward devia. tion is greatest on the opposite side. The phenomenon suggests a gravitational tide produced by the Clouds, but the effect appears to be about two orders of magnitude too large for a simple gravitational explanation. There may be an analogy with cases reported by $\mathrm{Z}_{\text {wicky }}{ }^{7}$ and others, which imply unexpectedly large interactions between galaxies.

The interpretation is less clear in the far wing of the line profile, beyond the region of the spiral arm peaks, where the intensity is very low. Here there is often a reversal in the curve of galactic latitude against radial velocity, from which the relation between height and distance is derived. This might be taken to indicate that the deviation of the equatorial surface from the principal plane decreases again beyond the region shown in the diagram. However, the reversal is associated with an increase in the angular width, and sometimes occurs at velocities which are too great to be fitted to a circular orbit model. Our interpretation is that the radiating gas in this case is relatively close to the Sun, and moving in non-circular orbits.

As well as giving the shape of the hydrogen concentrations, these observations can provide the point-by-point density distribution of neutral hydrogen throughout the galaxy. The detailed distribution has not yet been fully worked out for the southern observations, but we have derived provisional results for the overall amount of neutral hydrogen in the galactic disk, and its mean radial distribution ${ }^{8}$. These have been compared with corresponding figures for the total mass given by Schmidt's recent model ${ }^{9}$.

The ratio of neutral hydrogen to the total mass increases on going outwards from the centre; it rises from a very low value in the nucleus to about 15 per cent at the Sun's distance, and 50 per cent at 14 kiloparsecs out. The gas is a major constituent in the outer regions, but the proportion of neutral hydrogen integrated over the whole galaxy is only about 2 per cent.

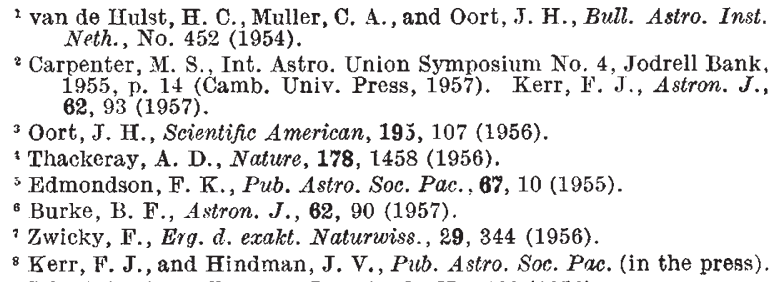

\title{
BIOGENESIS OF NATURAL PRODUCTS
}

\begin{abstract}
A SYMPOSIUM on "Biogenesis of Natural Products", arranged by Section B (Chemistry) of the British Association and held on September 6 in Dublin, was introduced by the president of the Section, Dr. J. W. Cook, who began by remarking on the appropriateness of the choice of Sir Robert Robinson as chairman. He recalled that Prof. J. N. Collie, of University College, London, himself a pioneer in the field, when introducing his successor, Prof. Robinson, remarked that his sort of chemistry was 'real' organic chemistry. This remark is even more recognizably true to-day.

Sir Robert opened his consideration of "Biogenesis of Structural Relations : the Organic Chemist's Contribution" by pointing out how organic chemists' interests in natural products have changed from
\end{abstract}

being an interest purely in structures to one concerned with the origins of such structures. His own pioneer work in the alkaloid field in 1917 indicated that structural relations do, in fact, reveal something of origins, although he realized that the final word is always with the biochemist. Recent powerful techniques such as the use of compounds containing isotopic carbon (carbon-14) have enabled the biochemist to test the predictions of the organic chemists. Their speculations have proved in some cases incorrect, but were correct in a surprisingly large number of cases.

$\mathrm{He}$ considered briefly the origins of fatty acids with an even number of carbon atoms from acetic acid units (containing two carbon atoms) and some of the other distinguishable biogenetic groups : the 
isopentane compounds (terpenes, steroids), the $\mathrm{C}_{6}-\mathrm{C}_{3}-\mathrm{C}_{6}$ groups and others.

Sir Robert's main thesis concerned the structural relations of alkaloids. The parallel series of pyrrolidine (five-membered ring) alkaloids, derived from the amino-acid ornithine, and the piperidine (sixmembered ring) alkaloids derived from the aminoacid lysine containing an extra carbon atom, had originally led to biogenetic theories since confirmed by tracer studies. The whole series of compounds of the isoquinoline sories can be correlated on the basis of biogenesis from phenylalanine and condensation with an aldehyde, followed by secondary reactions of methylation and oxidation. An attempt to do this in the laboratory in one case led to an alkaloid, then unknown, but discovered many yoars later in a Queensland tree. Adding a further carbon atom to the isoquinolines formed from two phenylalanine units leads to the berberine group, and hence by rearrangement to the sanguinarine group. Even morphine and related alkaloids, despite superficial appearances, can be derived (hypothetically so far) by chemically probable ring-closures in this series. Such considerations have led to a revision of the original formulxe of both berberine and morphine.

A similar series of alkaloids can be distinguished based on tryptophan instead of phenylalanine. Harmine is a strict analogue; others, such as strychnine, cinchonamine, quinamine and cinchonine, can all be related to a 'para' berberine type on the basis of a ring-fission postulated by R. B. Woodward. Thus, strychnine and quinine, despite enormous superficial differences, are biogenetically closely related. These ideas havo again been fruitful in leading to correct structures for emetine and ajmaline.

Sir Robert said that Nature is very clever; but listening to his survey gave the impression that some chemists at least are not far behind.

Prof. A. J. Birch (Manchester) discussed the use of isotopically labellod compounds-mainly $1^{1-11} \mathrm{C}$-acetic acid, ${ }^{14} \mathrm{C}$-formic acid and ${ }^{14} \mathrm{CH}_{3}$-methionine-for testing hypotheses based on structural analogies, using moulds as the exporimental media. Moulds were chosen because of their ease of growth, the ease of obtaining incorporations, the variety and high yields of some of the compounds produced and their intrinsic interest as antibioties and antifungal agents. A postulated biosynthesis of griseofulvin by headto-tail linkage of seven acetic acid units, and appropriate cyclizations, has been confirmed by reference to the degradations of the isotopically labelled substance derivod from $\mathrm{CH}_{3}{ }^{14} \mathrm{CO}_{2} \mathrm{H}$. It was shown that, in some cases at least, C-methylation can occur from methionine or formic acid in the same way as can $\mathrm{O}$-, $\mathrm{S}$ - or N-methylation. Among the substances being investigated are terramycin and methymycin. Extensions to terpene biosynthesis were mentioned, and it was shown that $\mathrm{HOCH}_{2} \mathrm{CH}_{2} \mathrm{C}(\mathrm{OH})\left(\mathrm{CH}_{3}\right) \mathrm{CH}_{2} \mathrm{CO}_{2} \mathrm{H}$ (mevalonic acid) is an irreversible intermediate in producing the terpene chains of mycophenolic acid and mycelianamide. Prof. Birch pointed out that the results obtained can be extrapolated to many products from higher plants, notably flavonoids and anthocyanins.

Prof. T. S. Wheeler (University College, Dublin) was not sure whether to serve a vegetarian diet for non-scientists or a meat diet for his colleagues; but he succeeded in producing a tasty well-balanced dish. He gave a summary of the chemical types related "to the flavones and flavanones which occur throughout higher plants. He pointed out that the $\mathrm{C}_{6}-\mathrm{C}_{3}-\mathrm{C}_{6}$ classification of Robinson is of fundamental importance. This turns out to be based probably on a $\mathrm{C}_{6}-\mathrm{C}_{3}$-unit with a $3: 4$-hydroxylation pattern (derived from shikimic acid) and a $\mathrm{C}_{b}$-unit (derived from acetic acid) with a $1: 3: 5$-hydroxylation pattern (giving 5:7-hydroxyls in the resulting flavonoid pigment). $\mathrm{H}_{\Theta}$ turned to consider the supporting evidence for these ideas, obtained by tracer experiments by Geissman and Neish and their collaborators on the biogenesis of quercetin in buckwheat. This work clearly establishes the sequence: shikimic acid $\rightarrow$ prephenic acid $\rightarrow p$-coumaric acid +3 (acetic acid) $\rightarrow a \mathrm{C}_{6}-\mathrm{C}_{3}-\mathrm{C}_{6}$ precursor.

Prof. Wheeler emphasized that many of the principles of chemical stability deduced in the laboratory clearly hold also in the biochemical world, and cited the equilibrium between chalcones and flavanones. The presence of a 5-hydroxyl group in the latter stabilizes them by hydrogen-bonding; similarly, a $2^{\prime}: 6^{\prime}$-dihydroxy flavanone can become isomerized (via the chalcone) to a more stable $2^{\prime}: 5$-dihydroxy flavanone. This may account for the lack of occur. rence of the latter type in Nature.

Prof. T. R. Seshadri (Delhi) put forward views on the part played by $\mathrm{C}_{8}$-units in natural products, particularly those found in lichens and in moulds. The typical $\mathrm{C}_{8}$-unit is that of orsellinic acid (2:4-dihydroxy-6-methylbenzoic acid), which enters into many depsides, and by secondary oxidation processes into depsidones. Some $\mathrm{C}_{8}$-units have an extra carbon atom, which may be $\mathrm{CH}_{3}, \mathrm{CH}_{2} \mathrm{OH}$ or $\mathrm{CHO}$, in the 3 -position, known to be a chemically active position. The presumption is that it is introduced. Prof. Seshadri considers that the most fundamental unit can be represented as 2:4-dihydroxy-6(hydroxymethyl) benzaldehyde, which can undergo various oxidations, reduction and condensation reactions leading to known depsides, depsidones, benzoquinones, anthraquinones and xanthones. He suggested that in some cases hydroxyl groups may have been reduced directly from the phenolic ring, and eited in support the reduction of the monotosylate of orsellinic acid with Raney nickel to obtain a good yield of 6-methyl salicylic acid. Further natural products, including stilbenes, tropolones and compounds related to gladiolic acid, can be produced by variants of the scheme. In the opinion of the present writer, this hypothesis amounts to a partial restatement of the acetic acid theory and its ancillary theory of the introduction of $\mathrm{C}_{1}$-1units, at a less-fundamental level, and is less satisfactory for a general correlation of the compounds. It also appears likely that mould tropolones arise from shikimic acid.

Dr. G. J. Popjak (Medical Research Council Radiopathology Research Unit, London) apologized quite unnecessarily for intruding on a gathering mainly of organic chemists, and explained that some fifteen years ago he had felt compelled to abandon his purely medical approach to atherosclerosis to find out something about the biochemical causes of it, notably the mechanisms of the biosynthesis of cholesterol. In the sequel it seems highly probable that the approach which he helped to pioneer is in fact the only logical one in attempting to cope with the disease. He explained that much of the chemistry was the result of his close collaboration with Dr. J. W. Cornforth. He discussed the earlier isotope work of Bloch and Rittenberg, which showed that acetic acid contributes the twenty-seven carbon 
atoms of cholesterol (fifteen from $\mathrm{CH}_{3}$, twelve from $\mathrm{CO}_{2} \mathrm{H}$ ). An amicable division of the field between the American and British groups has now led to an elucidation of the sources of all the individual carbon atoms in cholesterol (that is, whether from $\mathrm{CH}_{3}$ or $\mathrm{CO}_{2} \mathrm{H}$ ). This result, taken in conjunction with a much earlier hypothesis of Robinson that the acyclic triterpene squalene folds, with loss of three carbon atoms, into cholesterol, has permitted the elucidation of the major stages in the biosynthesis. A further great contribution has recently been made by Dr. K. Folkers and his group at Merck and Co., who have shown that the biochemical origin of the isopene unit is mevalonic acid $\left(\mathrm{HOCH}_{2} \mathrm{CH}_{2} \mathrm{C}(\mathrm{OH})\left(\mathrm{CH}_{3}\right) \mathrm{CH}_{2} \mathrm{CO}_{2} \mathrm{H}\right)$, which loses its carboxyl group in the process of incorporation. The overall process seems to be : acetic acid $\rightarrow X \rightarrow$ mevalonic acid $\rightarrow Y \rightarrow$ squalene $\rightarrow$ lanosterol (a $4: 4:$ 14-trimethyl steroid) $\rightarrow$ a $4: 4$-dimethyl steroid $\rightarrow$ zymosterol (a $\mathrm{C}_{27}$-steroid with $8: 14$ - and 24-double bonds) $\rightarrow$ desmosterol (5- and 24-double bonds) $\rightarrow$ cholesterol (5-double bond). The natures of some of the intermediates, notably $X$ and $Y$, and the exact mechanisms of some of the processes are still not at all clear.

The general impression left by the symposium was of rapid advance and a new air of certainty replacing somewhat misty speculations. However, it is clear that a pencil and paper are still very useful tools to produce useful and fruitful suggestions, if not proofs. Little time was left for discussion, and Sir Robert Robinson regretfully closed the symposium after thanking the speakers.
A. J. Birch

\section{CONSTITUTION AND CARE OF THE INFANT ANIMAL}

$\mathrm{S}^{\mathrm{B}}$ ECTIONS I (Physiology and Biochemistry) and M (Agriculture) of the British Association held a joint session during the recent meeting at Dublin on the subject of the infant animal. Prof. R. A. McCance opened the meeting with a general paper on "The Physiology of the Newborn Animal". He began by pointing out what a tremendous physiological upheaval birth must be, and then alluded briefly to the difficulty of finding one's way through the perplexing maze of species and developmental differences to the great general principles of infant physiology. He proposed, nevertheless, to try to do so, and began by saying a few words about the large volume of fluid outside the cells of all newborn animals. There may be some association, he suggested, between this and the anabolic impulse so characteristic of the foetus and newborn. Further expansion of the extracellular fluids by experimental means has in his experience materially reduced the catabolism of tissue protein. He then dealt with the ways and means by which the newborn animal is prepared by Nature to withstand the infections to which it is inevitably exposed the moment it leaves the sheltered world in utero. He traced out the path by which the macromolecules of $\gamma$-globulins and other proteins reach the plasma of the developing organism in the various species, and directed attention to some of the effects of this protein transfer, apart altogether from the establishment of passive immunity. $\mathrm{H}_{\theta}$ spent the rest of his time in discussing aspects of homoostasis. $\mathrm{H}_{\Theta}$ showed, for example, how unstable the body temperature can be in the newborn period, and referred to some of the factors responsible for its maintenance. He took as his examples the poikilothermic newborn rat, the piglet and the human infant, and compared and contrasted them. He then went on to deal with the response to a lack of oxygen, and began by referring to the excellent work which has been done on the subjects of cold and anoxæmia by the great pioneers, such as Harvey, Boyle and particularly Edwards. He made the point that, although there are great species differences, all newborn animals are very tolerant of anoxia. This is due, he believes, to the ability of the newborn animal to utilize anaerobic glycolysis to maintain the structure and function of its essential organs, particularly of its brain. The survival time in nitrogen is largely conditioned by the state of development of this organ, but any study confined to the survival-time tells one nothing about the complex respiratory and cardiovascular changes induced by a lack of oxygen in the first and final days of life. Anaerobic metabolism, he pointed out, utilizes the resources of the body wastefully and leads to the production of potentially dangerous end-products. It also produces less heat. This tends to reduce body temperature, which slows down metabolism all over the body, mitigates to some extent the dangers of the wasteful anaerobic glycolysis, and is an important element in prolonging the lives of anoxic newborn animals, including, in all probability, man. If this is so, resuscitation of the cold blue infant should begin with glucose to provide fuel for glycolysis and oxidation, pass on to re-oxygenation and be followed by warmth only. when oxygenation has become effective.

Prof. McCance then passed on to the subject of food, growth and renal function. This last is generally reckoned to be poor by adult standards in the new. born period, yet the concentration of urea in the serum is normal at birth and remains so. Few have seen the extent or significance of this paradox, but investigations carried out at Cambridge have explained it. The power of growth, it would appear, may be far more important than the operation of the kidney in maintaining the concentration of urea in the body fluids within normal limits. 90 per cent of the protein nitrogen in the diet of a rapidly growing animal may be utilized for the synthesis of tissue protein and only 10 per cent of it appear as urea and other end-products to be excreted by the kidney.

The weakness and helplessness of the infant have been a constant theme with philosophers. Helpless it may be in that it cannot stage its own come-back from an abnormal situation -it has to be brought back. But many of the signs of weakness are really marks of strength, if by strength is meant the ability to survive. The secret of handling and caring for the newborn is to make the most use of the strength with which it has been endowed by Nature, and if its stability has been upset, to restore this physiologically and not in some haphazard way which may seem the obvious one at first sight.

The next two speakers dealt with more specialized branches of the subject. Mr. G. A. Lodge, of the Rowett Research Institute, read a factual paper on 